Service social

\title{
La théorie et la construction des convictions en travail social
}

\section{Richard Zúñiga}

Volume 42, numéro 3, 1993

Perspectives théoriques

URI : https://id.erudit.org/iderudit/706630ar

DOI : https://doi.org/10.7202/706630ar

Aller au sommaire du numéro

Éditeur(s)

École de service social de l'Université Laval

ISSN

1708-1734 (numérique)

Découvrir la revue

Citer cet article

Zúñiga, R. (1993). La théorie et la construction des convictions en travail social. Service social, 42(3), 33-54. https://doi.org/10.7202/706630ar

\section{Résumé de l'article}

Le travail social est habituellement aux prises avec une rhétorique qui divise la réalité de l'action professionnelle en " théorie » et " pratique », et qui renvoie la première aux limbes du non-pertinent: « la pratique » semble pouvoir vivre sans elle. Un facteur qui contribue possiblement à cette exclusion de la théorie du monde de la quotidienneté professionnelle pourrait être l'identification qu'on en fait avec les discours de légitimation de la Science, de l'État et de l'Individu - discours qui renvoient le travail social à des structures de pouvoir qu'il respecte à distance, tout en s'en méfiant. Les perspectives constructivistes offrent des stratégies de décodification qui illuminent ce rapport aliéné d'une profession avec la recherche de sa propre rationalité. 


\title{
La théorie et la construction des convictions en travail social
}

\author{
Ricardo ZÚÑIGA \\ Professeur \\ École de service social, Université de Montréal
}

La théorie en travail social s'enracine dans des conceptions du social qui la précèdent et qui l'encadrent et l'orientent, et elle s'insère dans des contextes d'action qui la réinterprètent en matière de convictions. Les justifications et les discours de légitimation ne vident pas la question théorique, dans la mesure où ils ne soulignent pas assez l'autonomie intellectuelle du praticien.

La perspective constructiviste illumine les conditions de production et $d^{\prime}$ utilisation des théories, et suggère des pistes pour la formation. En fixant son regard sur le processus même de la structuration et de la formulation de l'action, elle permet une lecture critique de la Science, de l'État et de l'Individu comme des discours de légitimation. Elle permet ainsi de comprendre comment ces discours peuvent priver le travail social de sa propre rationalité.

\section{PARLER DE THÉORIE SANS FAIRE PARTIE DU PROBLÈME...}

Dans les milieux de pratique, le mot « théorie » a des connotations négatives. Le théorique est opposé au pratique, au réel et au réaliste, tout 
comme pour un praticien le théoricien désigne plutôt un autre, étranger, qu'un partenaire dans une action commune. Pour le praticien, la théorie semble liée aux souvenirs de formation, à l'apprentissage formel qui a précédé sa pratique professionnelle.

Les milieux de formation, quant à eux, ne sont pas plus libres d'idéologie. La théorie est située dans le respect des traditions intellectuelles reçues, en référence à des maîtres de pensée. La théorie fait ainsi plus partie de la culture scientifique universelle que de la construction personnelle. Le " cadre théorique » repose sur des citations, et doit répondre à la question : "connaît-il bien la littérature? " plutôt qu'à la question: " est-il un penseur original ? ». Selon l'expression mordante de Miguel de Unamuno, les citations constituent l'échafaudage pour la construction d'une pensée personnelle, mais la plupart des penseurs craignent $d^{\prime}$ enlever cet échafaudage après avoir fini la bâtisse, par peur qu'elle n'exhibe sa pauvreté et ne s'écroule par sa faiblesse. La formation des praticiens peut se limiter à s'assurer que les étudiants " connaissent » la théorie - non pas qu'ils I'utilisent et, encore moins, qu'ils la produisent: le respect de la théorie la prive paradoxalement de sa juste place dans la créativité et l'affirmation autonome de l'étudiant et du futur praticien.

Les deux milieux se reportent au sens habituel donné à la théorie. Pour tous les deux, il semblerait qu'il faille placer au centre du concept un «noyau dur»: au sens strict, la théorie serait « un ensemble de propositions enchaînées les unes aux autres permettant de déduire des conséquences qu'il est en principe possible de confronter à la réalité » (Boudon et Bourricaud, 1982: 617). Ce noyau semble faire appel à l'opérationalisation des concepts, à la formulation d'énoncés vérifiables, à une capacité de générer des affirmations prédictives, elles aussi vérifiables. Les débats scientifiques ne remettent pas en question ce noyau, qui est largement consensuel, et se concentrent sur l'évaluation comparative des théories.

Mais les débats sur la théorie ont aussi intégré progressivement une préoccupation pour mieux comprendre la place de la théorie dans les enjeux de l'action sociale; cette préoccupation a mené à accepter que la théorie implique une insertion sociale qui l'affecte, qui la pénètre: cette insertion est son caractère associé de paradigme.

Le terme lui-même véhicule l'ambiguïté de sa polysémie. Masterman (1974) signalait la multiplicité des sens du terme dans I'œuvre de Kuhn : il incluait les réussites scientifiques qui dominaient un champ de connaissances, les mythes et les croyances dans lesquels trempait ce champ, la tradition intellectuelle dans laquelle il s'insérait. II incluait aussi une analogie exemplaire, une métaphore ou une image structurante et une perspective épistémologique. La dissension se déclenche entre ceux qui voient théorie et paradigme comme une totalité indissoluble et ceux qui rejettent 
le concept de paradigme vers la périphérie de la théorie: "Si les croyances, [visions du monde] et idéologies jouent un rôle $d^{\prime}$ inspiration dans la production des paradigmes et des théories, il n'en résulte pas que les théories scientifiques soient assimilables à des idéologies" (Boudon et Bourricaud, 1982: 108).

Le débat actuel ne fait que récupérer une longue histoire. La racine grecque du concept de "théorie " signifie d'abord contemplation d'une unité cachée sous la multiplicité apparente du réel. Cette unité est faite de la proximité, l'affinité et la participation de l'esprit humain à la rationalité intrinsèque de toute réalité - ce qui constitue l'hypothèse fondamentale de la philosophie grecque (Gadamer, 1984 : 17-18). Dans cette conception, $c^{\prime}$ est la réalité elle-même qui, tout en étant multiple et fragmentaire pour le regard superficiel, est unifiée dans son essence, et donc apte à être comprise, "saisie», maîtrisée. C'est depuis la Renaissance que la conception de la théorie est renversée et qu'elle évoque un processus de construction de cette unité par l'esprit, une construction exploratoire, condamnée à chercher sa propre vérification. La théorie devient ainsi une construction de l'esprit parmi d'autres, et ces liens avec ces autres constructions croyances, superstitions, significations vulgaires - deviennent un objet d'étude. Dans les sciences sociales, les "paradigmes » de Kuhn trouvent des concepts équivalents dans les "présupposés d'arrière-plan" et les "présupposés de domaine » de Gouldner (1970), qui représentent les contextes culturels et le champ spécifique de connaissances sur lesquels se greffe une théorie - si elle réussit à développer une coexistence pacifique avec eux. Ils se relient aux métaphores dominantes grecques, chrétiennes et modernes des théories du changement social de Nisbet (1969); ils se relient, enfin, aux «mythes fondateurs " des sciences sociales de Claval (1980):

$C^{\prime}$ est peu à peu, en interrogeant les maîtres de la sociologie, de I'histoire, des sciences politiques ou de l'ethnologie, qu'il nous est apparu nécessaire de nous arrêter à leurs présupposés et aux mythes fondateurs par lesquels leur pensée a acquis sa portée. $C^{\prime}$ est dans la dialectique de ces philosophies sociales que résident les difficultés, les incertitudes, mais aussi l'attrait des disciplines de la société (Claval, 1980 : 10).

\section{PRATIQUES ET DISCOURS: JUSTIFICATIONS ET LÉGITIMATIONS}

La pratique du travail social se structure et s'organise dans I'action, mais les explications de la dynamique de cette action restent souvent dans la périphérie de la vitalité des pratiques. Pourquoi intervient-on? Qu'est-ce 
que le praticien trouve dans son intervention pour alimenter son enthousiasme? Comment expliquer cette intervention par ses effets, mais aussi par son dynamisme et par sa vitalité?

La recherche de la vitalité des pratiques peut commencer par l'analyse des discours que celles-ci génèrent. Nous appellerons justifications les raisons qu'elles offrent pour expliquer une intervention, un comportement ou une stratégie; nous appellerons légitimations les justifications qui s'appuient sur des raisons reconnues comme étant socialement (scientifiquement et politiquement) valables. Prenons un exemple: dans le cadre $d^{\prime}$ 'une intervention en contexte d'autorité, le praticien justifie un jugement de dangerosité à partir de son expérience professionnelle, et il légitime sa recommandation en se référant à l'article pertinent de la loi. Dans les deux cas, les raisons apparaissent comme des réponses inévitables à ceux qui mettent en question les pratiques; toutes les deux prennent la forme défensive "j'ai fait comme ça, parce que..." .

Dans les deux cas, l'explication commence dans la compétence verbale du praticien, dans sa capacité de relier une action à un discours. Dans le cas de la légitimation, elle exige une compétence supplémentaire, celle de connaître les «bonnes raisons " pour justifier l'action. Mais ces discours rendent-ils compte de la totalité de l'action?

Protégées par les rationalités officiellement sanctionnées, les pratiques sociales d'intervention se sont cramponnées aux discours de légitimation. Les pressions sociales et les filtres sociopolitiques des connaissances ont limité la recherche d'une compréhension des enracinements des choix d'action à une stratégie défensive de l'intervenant. Pour lui, " faire de la théorie" se limite à citer les orientations et les priorités des gouvernements dans la "problématique», à citer les études scientifiques les plus diffusées dans le "cadre théorique " et à proposer l'utilisation d'instruments reconnus comme valides et fiables dans la «méthodologie ». Ce traitement institutionnalisé de la théorie ne souligne pas assez le caractère personnel de la connaissance, l'ancrage nécessaire de l'action dans les convictions de l'acteur, et des convictions dans les certitudes qui lui servent de fondement, soient-elles des vérités scientifiques, des hypothèses jugées probables ou plausibles, ou encore des espérances - qui sont les formes de certitudes les plus subtiles. Quand un politicien nord-américain invite à accepter le fait que la situation " va s'aggraver avant de s'améliorer ", et quand il affirme voir "la lumière au bout du tunnel », il entraîne des sociétés (censées être rationnelles) dans des entreprises qui relèvent autant de l'espérance que de la foi, les plus opposées qui soient à la rationalité de laquelle elles se réclament. Convictions et certitudes, foi et espérance sont les ingrédients vivants d'une théorie-en-action, du dynamisme et de la passion d'une action - même la plus soigneusement éloignée de l'acteur par des soucis d'objectivité et de scientificité. 


\section{LE TRAVAIL SOCIAL ET L'EMPRISE DES DISCOURS DE LÉGITIMATION}

Les discours habituels en travail social se formulent en respectant les paramètres du social accepté et prôné. II est entendu que les discours fondés sur des convictions religieuses ou morales ne sont plus à la mode; et il est toujours risqué de parler en termes de convictions politiques partisanes. II reste le choix de parler en termes de discours officialisés de légitimation. Trois discours dominent le champ.

D'abord, la science, comme fondement du travail social, semble fournir une rationalité considérée comme indiscutable. Selon ce discours, il y aurait un défi de compréhension, en arrière-plan de chaque situation sociale problématique, et $c^{\prime}$ est cette compréhension interprétée qui pourrait fournir les hypothèses à vérifier par une méthode transparente parce que parfaitement rationnelle: «la méthode scientifique»-au singulier. De la connaissance vérifiée à la "science sociale appliquée " il n'y aurait qu'un petit pas à faire. "Appliquer " paraît être une déduction simple à partir d'un principe général vers une action concrète, qui serait son corollaire évident. La science serait ainsi appliquée par une opération logique plutôt que par un choix parmi des actions alternatives, choix qui ne dérive pas de la connaissance pure, mais de son insertion dans l'univers des jugements de valeur et des choix des acteurs.

Un deuxième discours reconnaît l'identification des problèmes sociaux comme un acquis collectif, de la société dans son ensemble ou des collectifs critiques dans son sein. Les problèmes fournissent un équivalent social à une objectivité matérielle: ils sont là, irréfutables. Pointer un problème fait appel au réalisme collectif pour valider l'évidence du problème et le corollaire de l'action correctrice. Le problème serait indiscutable; la solution serait évidente. Le travail social agirait sur ces défis identifiés collectivement, qui seraient aussi les garants de la pertinence sociale de l'intervention.

Un troisième discours fonde l'action d'intervenir sur la demande qui est adressée au travail social par un client présent ou futur. Le travail social répondrait ainsi aux personnes et aux collectifs qui auraient recours aux compétences et aux ressources disponibles dans les services sociaux. L'objectivité serait garantie par la correspondance entre la demande formulée, le besoin qui serait à son origine et le service offert.

Ces définitions de la raison d'être de l'action éclairent les questions de départ, mais ne les épuisent pas. On ne comprend pas mieux pourquoi il faudrait intervenir, ni pourquoi on intervient dans les faits. Les liens entre le travail social et la science sont aussi ténus que ceux entre l'activité quotidienne dans les services sociaux, la recherche scientifique existante et les enseignements des centres de formation. Les définitions des problèmes sociaux traduisent mieux les priorités de l'État et des filières qu'il 
finance ou contrôle que les actions qui en découlent, soient-elles gouvernementales, "parapubliques» ou formellement autonomes. Le discours officiel selon lequel les agences ne feraient que répondre aux demandes des usagers ne s'arrête pas assez sur l'analyse des mécanismes de filtrage sélectif et de réinterprétation systématique par lesquels les demandes de services sont transformées selon les priorités et les possibilités des services.

Les trois réponses ont cependant des traits communs: elles s'appuient sur une confiance en la rationalité de la modernité. En tant que Raison, État et Individu, elles ne reflètent que trop l'effort idéologique de réduire les questions sociales du "pourquoi agir?" aux réponses formelles des "parce que " limitées aux raisons données comme justification et comme légitimation, réponses en fonction de la nécessité externe plutôt $q u$ 'en fonction de la décision d'agir. "...Le social vient ainsi à désigner $d^{\prime}$ abord ce travail d'organisation rationnelle de l'être-ensemble et de la solidarité »; [...] "les sociétés modernes peuvent ainsi aspirer à la transparence $d^{\prime}$ un social enfin épuré de tout ce qu'il contient de symbolique, d'ambigu, de contradictoire et d'hétérogène » (Renaud, 1990: 29). On ne peut que voir avec préoccupation comment les discours de légitimation limitent la théorie à un discours de communion avec le social officialisé, et libèrent le praticien de la responsabilité de découvrir et de construire la théorie qui expliquerait son action, qui serait, elle aussi, un produit de son agir.

Parmi les mouvances intellectuelles qui cherchent à estomper les frontières de la rationalité instrumentale, socialisée et officialisée, pour ainsi affaiblir sa revendication de la totalité du champ de la raison d'être de l'action sociale, le constructivisme occupe une place potentiellement prometteuse.

\section{LA PERSPECTIVE CONSTRUCTIVISTE}

Une définition approximative du constructivisme pourrait souligner sa façon caractéristique de reprendre les questions sur le savoir et le connaître. Son intérêt n'est pas de se concentrer sur le "qui connaît? " de la psychologie cognitive ni sur le «qu'est-ce qu'on connaît? » de l'épistémologie philosophique, mais plutôt sur " $C^{\prime}$ est quoi, la recherche de la réalité? » (Watzlawick, 1988: 10). Cette dernière orientation situe le sujet et l'objet, l'esprit et la réalité dans le processus actif de construction d'une connaissance en tant que rapport social.

À partir de von Glasersfeld (1991), Larochelle et Désautels (1992, 25-27), on peut résumer le constructivisme selon quatre idées maîtresses. 
Son fondement est le scepticisme, qui souligne l'impossibilité de valider la connaissance acquise par l'expérience avec celle issue d'une source de connaissance autre. Les conjonctures historiques ajoutent la théorie instrumentaliste comme un moyen de sauver la religion - et, après elle, le politique -, en limitant le scientifique à la méthode, en laissant les explications globales et les choix d'action sociétale (projets de société, orientations, priorités) aux théologiens et aux politiciens. La troisième idée est la conscience du caractère construit des concepts, le caractère "fait " des faits scientifiques, qui permet à Knorr-Cetina de se référer à la science comme à une «manufacture des connaissances» (1981). La quatrième idée est l'évolution comprise dans son sens original, comme un processus d'extinction sélective des variations les moins viables, et non pas comme une orientation divine vers un but préétabli. Les quatre idées maîtresses ensemble soulignent le rôle d'un sujet actif, créateur de significations et d'un savoir actif; un sujet aux prises avec un monde réel dans lequel il essaie de fonctionner et de se réaliser comme un sujet viable et aussi capable de rapprocher le réel à sa vision d'un possible.

Dans cette perspective, ni la science, ni aucune connaissance ne cherche à produire une copie dans l'esprit d'une réalité qui lui serait extérieure. Dans des termes anglais succincts, la science ne vise pas un match, la production d'une correspondance entre une réalité matérielle et sa représentation, une copie conforme d'une réalité-objet. Elle se contente d'un fit - c'est-à-dire d'une connaissance appropriée aux données disponibles, une adaptation de la pensée et de l'action à l'information accessible. Dans l'exemple imagé de von Glasersfeld, les cambrioleurs ont démontré qu'on peut ouvrir une serrure même sans avoir la clé précise: leur passe-partout est une approximation suffisante à la forme de "la vraie clé », s'il réussit a déclencher le mécanisme de la serrure. Ils n'ont pas besoin de « comprendre» la serrure: il leur suffit de vérifier l'efficacité du passe-partout.

Cette stratégie cognitive qu'est le constructivisme rompt avec les dilemmes où s'opposent sujet et réalité, subjectivité et objectivité. Comme Piaget, le constructivisme accepte que "I'intelligence organise le monde en s'organisant elle-même " (von Glasersfeld, 1988: 27); à l'instar de Maturana, il souligne que "tout faire est un connaître " et que "tout ce qui est dit est dit par quelqu'un» (1984: 13). C'est aussi l'épistémologie évolutionniste de Campbell (1988 a, b), cette épistémologie qui est aussi une critique sociale de la construction des sciences. Les antécédents scientifiques directs la relient à Popper, à Kuhn et au questionnement du scientisme dans ses formes les plus triomphalistes: " En un sens, faire de l'épistémologie aujourd'hui, c'est n'en pas croire un mot» (Nadeau et Désautels, 1984 : 16). Les racines philosophiques indirectes sont celles de 
toute I'histoire de la philosophie; les liens directs la relient aux travaux de Schutz sur la construction des réalités multiples (Schutz, 1962, 1973), à l'influence marquante des disciples de Schutz, comme Berger et Luckmann (1967), et aux analyses de la construction culturelle du sens commun (Geertz, 1983).

\section{LE REGARD CONSTRUCTIVISTE SUR LES DISCOURS DE LÉGITIMATION}

Le constructivisme permet de revenir de façon critique et avec un regard nouveau sur les discours de légitimation de la Science, de l'État et de I'Individu. Ce retour, même s'il retarde notre analyse du problème spécifique de la théorie en travail social, est exigé à la base même de notre argumentation: le travail social ne pourra construire sa pensée sans exorciser préalablement des fantasmes qui paralysent son autonomie.

\section{Science et constructivisme: les fleurs et la botanique}

Le constructivisme s'enracine bien dans l'évolution post-popperienne de la philosophie des sciences. L'incorporation de la critique du réalisme naîf et de l'empirisme radical ainsi que du "vérificationnisme » et de la conception d'une science censée être pure devient un acquis de l'analyse du discours de justification des sciences. La méthodologie intègre aux panégyriques de "la méthode» les avertissements de l'épistémologie:

Nous avons évoqué précédemment l'avantage qu'avait représenté l'acquis de la conscience dans le processus d'évolution - à savoir, l'avantage de pouvoir transférer le risque de mort de l'individu à celle de l'hypothèse qu'il fait. Ce passage de la réalité matérielle au domaine de la pensée est sans doute un des plus importants acquis de l'histoire de l'évolution. Mais on ne doit cependant pas oublier les pièges liés à ce progrès. Toutes les erreurs désastreuses qui en ont résulté ont la même origine: une vérification dans le domaine de la pensée est prise pour une vérification réelle et réussie dans le monde concret (Riedl, 1988: 91).

La sociologie des sciences incorpore ainsi une perspective qui regarde la science comme une construction sociale, culturellement enracinée et encadrée:

La thèse en discussion est celle ci : les produits de la science sont des constructions spécifiées par leur contexte, qui portent la marque de la contingence situationnelle et de la structure des intérêts du processus par lequel ils sont générés; ils ne peuvent pas être 
adéquatement compris sans une analyse de cette construction. Ceci signifie que ce qui se passe dans le processus de construction marque les produits que nous obtenons. II signifie, aussi, que les produits de la science doivent être perçus comme étant très structurés internement par le processus de leur production, indépendamment de la question de leur structuration externe par une correspondance ou manque de correspondance avec la réalité (Knorr-Cetina, 1981: 5; trad. RZ).

Dans une manufacture, comme dans toute autre manufacture, un artisan travaille le bois, et le meuble en résultant reflète autant le bois que l'artisan. Les questions sur l'objectivité ou sur la rigueur qui seraient propres aux sciences deviennent les équivalentes des questions sur ce que serait le meuble sans le bois et ce que serait le meuble sans I'artisan... La perspective aboutit à un "programme fort ", dans lequel la science est analysée avec une «impartialité » qui la considère comme équivalant à toute autre construction culturelle:

Le programme fort. Le sociologue s'intéresse à la connaissance y compris à la connaissance scientifique - en tant que phénomène normal. II en donnera donc une définition nettement différente de celle du profane ou du philosophe. Pour lui, la connaissance sera, non pas la croyance vraie, mais tout ce que les hommes considèrent comme connaissance, c'est-à-dire ce qu'ils croient en toute confiance et dont ils vivent. Le sociologue s'intéressera en particulier aux croyances tenues pour acquises, à celles qui sont institutionnalisées, ou à celles que tel ou tel groupe revêt d'un caractère d'autorité. Bien sûr, il faut distinguer la connaissance de la simple croyance. Pour cela, nous réserverons le mot connaissance à ce qui est accepté collectivement, et nous mettrons au compte de la simple croyance ce qui est individuel ou singulier (Bloor, 1976; trad. Pandore: 5).

$\mathrm{Si}$ "le programme fort " repose sur l'affirmation d'un relativisme radical, il ne constitue cependant pas une négation de la démarche scientifique : il exige seulement que celle-ci soit revue comme une activité constante de révision et de reformulation. II représente néanmoins la polarité opposée à celle des défenseurs de la science comme vérité objective:

Dans le $10^{\mathrm{e}}$ chapitre de Modeste Mignon Honoré de Balzac écrit une phrase philosophique: "La botanique est venue après les fleurs ». Lorsque j'ai lu cette phrase à ma fille, étudiante de cégep, elle s'est écriée: "Mais Papa, c'est évident. II n'y a pas besoin de le dire. " À quoi je répondis: "Peut-être, mais il y a des gens que diraient que les fleurs sont des "constructions sociales de la communauté des botanistes" . C'est-à-dire, que d'abord il y eut des botanistes, après des fleurs. Que dis-tu de ça? » La réponse de ma 
fille fut rapide: "Évidemment, ces gens-là n'ont jamais pensé qu'on a trouvé des fleurs fossiles, mais pas des botanistes fossiles.»

Ce dialogue résume le débat entre les réalistes, comme ma fille, Aristote, Galilée, Newton, Darwin, Planck, Einstein et autres, et les philosophes et sociologues des sciences qui, de Ludwick Fleck à Thomas Kuhn, Paul Feyerabend, Barry Barnes, David Bloor, Bruno Latour et plusieurs autres, ont réussi à combiner l'idéalisme philosophique avec la sociologie de la connaissance et l'anthropologie. Ce qu'il y a d'original dans leur doctrine c'est que c'est une sorte d'idéalisme collectiviste [...]. La question est de savoir si l'idéalisme collectiviste est vrai ou faux (Bunge, 1991: 41).

Les défenseurs d'une troisième voie, plus spécifiquement méthodologique, doivent d'abord essayer de surmonter l'acrimonie du débat épistémologique actuel, entre la radicalité sociologique et la certitude tranquille de Mlle Bunge, cela tout en acceptant et en défendant qu' «il n'existe à proprement parler aucune théorie de la scientificité qui fasse consensus à I'heure actuelle» (Nadeau, 1991: 34). Pour Nadeau, il y a l'image médiatique de la science, la science présentée comme le moteur unique du développement économique et de tout progrès technique, la science qui se justifie par la R-D (recherche et développement). Il y a, aussi, I'image sociologique de la science-mythe tribal contemporain, la science du roman contemporain à la Eco, qui va du constructivisme radical ironique du moine-détective protagoniste du Nom de la rose (1980) à l'ironie encore plus radicale du Pendule de Foucault (1990), moquerie de la possibilité de distinguer entre les postulats de l'esprit et une réalité qui lui serait extérieure. Les deux laissent encore de la place pour une image épistémologique de la science, une activité de pensée ouvertement normative, dans laquelle la philosophie peut se situer «dans le processus réflexif lui-même, c'est-à-dire dans la quête des arguments corrects et dans la critique des affirmations sans fondement " (Nadeau, 1991 : 34). Cette troisième voie permet de mieux comprendre les deux autres, et d'éloigner les craintes - injustifiées - qu'elles déclenchent: la politicisation autoritaire d'une science officielle d'État ou la « destruction sociale de la réalité »! (Hollis, 1982). Elle pourrait aussi aider à comprendre la distance inquiétante entre les perspectives méthodologiques qui se réclament des conceptions opposées. Les cours des «méthodes quantitatives " coexistent avec ceux des "méthodes qualitatives", mais ils ne cherchent pas une logique commune, et l'épistémologie gagne des cartes de citoyenneté là où toute la structuration intellectuelle semblait dépendre seulement des «méthodes « et de la «méthodologie« . À la découverte étonnante que nous parlons en prose pourra s'ajouter bientôt celle que nous sommes tous des abonnés de l'épistémologie. 


\section{Problèmes sociaux, soucis d'État}

L'analyse constructiviste des problèmes sociaux mène rapidement à souligner leur caractère de construits d'État: la validation du savoir par un programme de parti, le vote et les sondages, et le mandat qui en découle. II est difficile de trouver une formulation plus frappante dans son honnêteté brutale que celle du Livre blanc sur la recherche scientifique:

En somme, la poursuite d'un idéal démocratique en matière de science et de recherche exige que soient respectés les droits et les aspirations de tous les intéressés. Au premier chef, bien sûr, les chercheurs eux-mêmes, avec leur légitime prétention au libre exercice de leur activité. Mais aussi, à des titres différents, l'ensemble des citoyens, dont la qualité de vie constitue une priorité non négociable, et l'État, cet interprète particulièrement autorisé des intérêts collectifs. [...] Dans ses démarches mêmes, l'activité scientifique est sans doute d'abord l'affaire des scientifiques et n'obéit qu'aux impératifs de la poursuite du vrai. Mais, replacés dans le contexte plus global de la vie collective, il est évident que la science et le pouvoir qu'elle confère ne sauraient être laissés aux mains des seuls scientifiques; la science concerne vraiment tout le monde et c'est pour cela que son développement doit tenir compte des priorités identifiées par l'ensemble des politiques de développement. En poursuivant un tel projet démocratique en matière de recherche scientifique, $c^{\prime}$ est de ces priorités collectives que le gouvernement entend se faire le défenseur et le promoteur auprès de la communauté scientifique québécoise (Québec, $1980: 17$; italiques RZ).

Lyotard, dans son rapport au Conseil des universités auprès du gouvernement du Québec, analyse ainsi cette science d'État:

$\mathrm{C}^{\prime}$ est depuis Platon que la question de la légitimation de la science se trouve indissociablement connexe à celle de la légitimation du législateur. Dans cette perspective, le droit de décider de ce qui est vrai n'est pas indépendant du droit de décider de ce qui est juste, même si les énoncés soumis respectivement à l'une et l'autre autorité sont de nature différente. C'est qu'il y a jumelage entre le genre de langage qui s'appelle science et cet autre qui s'appelle éthique et politique: I'un et l'autre procèdent d'une même perspective ou si l'on préfère d'un même "choix ", et celui-ci s'appelle l'Occident [...]... savoir et pouvoir sont les deux faces d'une même question: qui décide ce qu'est savoir, et qui sait ce qu'il convient de décider? La question du savoir à l'âge de l'informatique est plus que jamais la question du gouvernement " (Lyotard, $1979: 20$; italiques RZ). 
$C^{\prime}$ est cette emprise gouvernementale sur la construction des problèmes sociaux qui fait dire à un chercheur :

Considérant le pouvoir politique énorme dont sont investis les appareils de gestion des problèmes sociaux, il est à craindre que la recherche sociale soit domestiquée au point qu'elle en vienne à participer aveuglément au projet de rationalisation et de structuration de l'action sociale (Lefrançois, 1990: 138).

\section{Le client souverain et les lois du marché}

La glorification du client-consommateur comme mesure ultime d'objectivité sociale peut illustrer les contributions controversées du constructivisme extrême aux débats scientifiques. Guba et Lincoln (1989) font une extrapolation logique frappante, qui fait de la sociologie de la connaissance un manifeste relativiste, et une déclaration de foi dans le caractère illusoire de la réalité, position extrême, embarrassante pour beaucoup de constructivistes, qui ont insisté sur le fait qu'ils analysent un processus de connaissance, et non pas la nature ultime de la réalité.

Nous croyons que le paradigme constructiviste est adéquat [pour remplacer le paradigme positiviste dans les sciences]. II ressemble peu à la science, surtout dans ses présupposés de base, qui sont presque à l'opposé de ceux de la science. Parce que du point de vue ontologique, il nie l'existence d'une réalité objective: il affirme, au contraire, que les réalités sont des constructions de l'esprit, et qu'il y a autant des constructions qu'il y a d'individus (même si plusieurs constructions seront partagées). Nous affirmons que la science est une de ces constructions. Nous sommes prêts à l'admettre au panthéon des constructions, mais à condition qu'on ne nous demande pas de l'accepter comme la seule vraie ou correcte. Et nous notons que si les réalités sont des constructions, il ne peut pas y avoir (sauf par attribution mentale) des lois immutables qui encadrent ces constructions, comme les lois de causalité (Guba et Lincoln, 1989 : 43-44; trad. RZ)

II faudrait souligner ainsi ce que le constructivisme $n^{\prime}$ est pas - au moins du point de vue de cet humble amateur. Le constructivisme n'est pas une nouveauté. II est un questionnement de longue date, alimenté par des courants de conscience critique toujours présents dans les sciences sociales. II n'est pas, non plus, une aventure irresponsable dans la philosophie - ni un idéalisme métaphysique, qui affirmerait allègrement que tout est illusion; il n'est pas non plus un relativisme cynique, qui resterait dans I'affirmation que «tout est relatif ». II n'est pas non plus un déterminisme antiscientifique ou antipolitique, une déqualification de toute connaissance scientifique par sa réduction à ses déterminants externes : 
" he who pays the piper calls the tune ; " chacun prêche pour sa paroisse ". Le constructivisme ne définit pas la science comme l'activité nécessaire pour obtenir des subventions ou des patentes, et n'identifie pas, non plus, la définition des problèmes sociaux aux stratégies électorales des partis en lutte. Mais, par ailleurs, le constructivisme n'accepte pas d'identifier les convictions qui animent les acteurs passionnés et engagés avec la validité des certitudes qui les guident. II ne permet pas, non plus, d'accepter les discours de justification et de légitimation comme une compréhension suffisante de la passion - intellectuelle autant qu'existentielle - de l'action sociale. Si la communauté scientifique, l'État et les clients sont objectivement des acteurs, aucun des trois $n^{\prime}$ est une garantie d'objectivité de la connaissance qui se construirait sous leur égide, aucun des trois n'est une explication en soi suffisante de la genèse et de la dynamique de I'action.

\section{CONSTRUCTIVISME ET TRAVAIL SOCIAL}

D'où proviennent les idées correctes? La question charnière de la praxéologie marxiste (Mao Tse Toung, 1972) hante encore l'authenticité intellectuelle du travail social. Pour le travail social, le besoin toujours présent d'une identité intellectuelle enracinée dans I'action quotidienne reste une aspiration refoulée, autocensurée. England a fait une analyse que nous partageons entièrement:

Les gens de bon sens (dont les travailleurs sociaux) ne se tapent pas la tête contre les murs sans bonne raison. Les bons travailleurs sociaux connaissent, de par leur expérience, la valeur de leur travail auprès de leurs clients. [...] Leur incapacité pour expliquer [cette barrière entre le cœur et la tête], entre vivre et comprendre, les place dans un dilemme; leur incapacité à communiquer leur expérience à ceux qui les critiquent est un fait sans issue, compte tenu de la structure habituelle du débat sur la nature du service social. Les critiques demandent une réponse " matérialiste », en termes des procédures et des effets mesurables - ce qui est tout à fait raisonnable dans le débat conventionnel. Les travailleurs sociaux essaient d'y répondre, mais ils le font dans les termes et les concepts usuels dans la profession. Mais l'expérience des uns et le langage des autres ne se rencontrent pas et ils ne changent pas, non plus. Et le travail social continue à tourner en rond (traduction paraphrasée de England, $1986: 4-5$ ).

Le chemin déblayé par England rejoint le cheminement des auteurs comme Schön (1991,1987 et 1983), Racine (1991) et St-Arnaud (1992), avec leurs analyses de la réflexion dans l'action; il trouve aussi des 
héritiers dans le travail social. Pour Goldstein, la révision de la construction des connaissances en service social doit reconnaître son caractère de mélange de théorie, analogie, sagesse et art (Goldstein, 1990: 41); pour lui, ce mélange reflète la variété des demandes et des pratiques, mais aussi l'échec des efforts de la recherche de légitimation dans le caractère de la pratique de service social censée être scientifique. Sa perspective analytique se rapproche davantage du constructivisme de Scott (1989), qui l'enracine dans l'œuvre d'England. Avec eux, elle accepte de reconnaître que "la compréhension de l'interprétation du monde du client est la caractéristique définitionnelle du service social» (Scott, 1989: 40) et que « chaque cas est une constellation unique des significations et des définitions possibles de la situation, par les différents acteurs, les réalités multiples de tous les participants impliqués - ainsi que celle du travailleur social » (Scott, 1989: 41). Elle s'éloigne d'England seulement pour remettre en question son choix du terme "intuitif " pour décrire la connaissance-dans-I'action du praticien et choisir la perspective de Polanyi sur la connaissance personnelle, la connaissance tacite qui anime l'action du praticien:

Nous arrivons ici au problème le plus crucial de la théorie de la connaissance. [...] J'ai essayé de démontrer que dans tout acte de connaissance il y a une contribution tacite et passionnée de la personne qui est en train de connaître ce qui est connu, et que ce coefficient $n^{\prime}$ est pas simplement une imperfection, mais une composante indispensable de toute connaissance. Toute cette évidence devient une démonstration de l'absence totale de fondement de toute connaissance, si nous ne pouvons pas défendre de tout coeur nos propres convictions, même quand nous envisageons la possibilité que nous ne pourrons pas les défendre (Polanyi, 1964: 312).

Si nous essayons de reprendre la perspective de Watzlawick et de ses collègues, nous pouvons comprendre le constructivisme comme une ouverture de l'étude de la dynamique intellectuelle, pour mieux inclure les convictions sociales, éthiques et politiques, qui dirigent toute action de société. Le défi de l'action sociale est celui d'agir sans pouvoir compter sur une source de certitude qui la protégerait de son caractère d'exploration dans le jamais complètement connu. Elle devra construire sa rigueur intellectuelle par son travail de réflexion critique et son honnêteté, qui est une reconnaissance du caractère faillible des fondements factuels de ses convictions.

... dans quel monde vit-on si on accepte que l'on construit entièrement soi-même sa propre réalité?

Avant tout, [...], on est tolérant. Si nous voyons le monde comme notre propre invention, nous devons admettre que tout un chacun 
en fait autant. Si nous savons que nous ne connaissons jamais la vérité, que notre vision du monde convient seulement plus ou moins, comment pourrions-nous alors considérer les visions des autres comme démentes ou mauvaises et rester attachés à la conviction manichéenne primitive «Qui n'est pas pour moi est donc contre moi "? Pour être capable de respecter la réalité que les autres inventent pour eux-mêmes, nous devons d'abord comprendre que nous ne savons rien tant que nous ne savons pas que nous ne connaîtrons jamais la vérité absolue. Et c'est seulement si les autres deviennent intolérants à l'égard de notre propre réalité, que nous pouvons alors - citons ici encore Karl Popper - « au nom de la tolérance, revendiquer le droit de ne pas tolérer l'intolérance ».

Ensuite, vivre dans un monde constructiviste, c'est se sentir responsable, au sens profondément éthique du terme, non seulement de nos décisions, de nos actes et de nos rêves, mais aussi, dans un sens beaucoup plus large, de la réalité que nous inventons chaque fois que nous faisons des prédictions qui se vérifient d'elles-mêmes. Dans un monde constructiviste, il n'est plus question de confortablement rejeter la faute sur les autres ou sur I'environnement (Watzlawick, 1988, 350-351).

Watzlawick va encore plus loin. La conscience des possibles, des alternatives est en soi une libération: c'est la possibilité de vouloir construire l'alternative visualisée.

À ce point-ci, nous pouvons dégager quelques affirmations exploratoires.

Une discussion de la théorie en travail social ne peut séparer la connaissance de l'action, le connaître de l'agir, et ne peut accepter qu'ils soient incarnés dans des acteurs différents, découpés l'un de l'autre (voir figure 1, p. 48).

Agir et connaître renvoient à un même monde ; «la pratique » et "la théorie " posent des questions complémentaires, inséparables. La théorie identifie la raison d'être de l'action, répond à la question "pourquoi agir? ", guide l'acteur dans les choix auxquels il est confronté dans son agir, et lui permet la prise cohérente des décisions. La pratique est l'action guidée par une pensée, et cette pratique fournit à l'acteur les éléments de nature à alimenter sa réflexion. Théorie et pratique progressent ensemble, se modifient mutuellement. La pratique développe progressivement sa capacité de comprendre le pourquoi et le comment de son effort pour produire des changements. La théorie s'enracine graduellement dans la vie active et incorpore à sa vision l'information qui lui est fournie par l'expérience.

Deux corollaires découlent de cette perspective d'analyse. Le premier est que la connaissance de la théorie en travail social ne peut être 
Figure 1

Le connaître et I'agir

\section{l'agir quotidien: qui connaît?}

les habitudes sociales,
l'éthique et les valeurs,
le politique et les choix de société

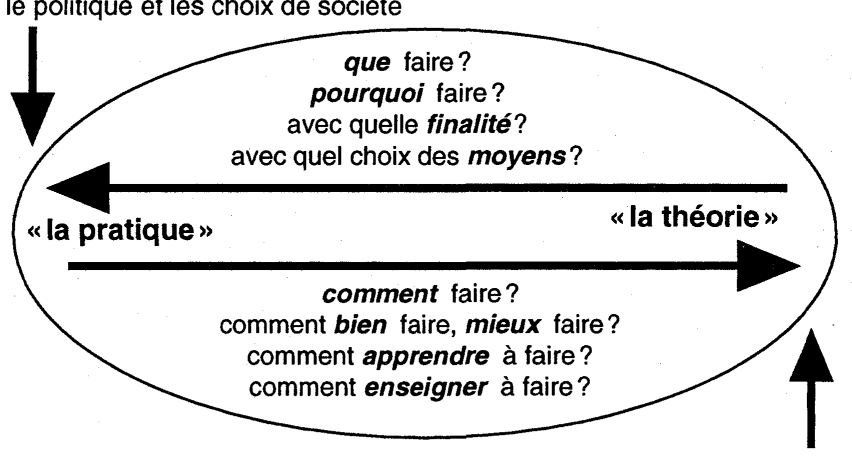

le connaître quotidien: qui agit?

la pensée sociale quotidienne, les "pris pour acquis " et l'institué,

les acquis des sciences et de la réflexion critique

comprise comme une abstraction préalable à l'intervention, apprise, reçue. II faut la comprendre comme une "théorie-en-usage », selon l'expression d'Argyris et Schön, comme la rationalité d'une action. La pensée théorique est toujours en élaboration, en développement continu, à la faveur de l'expérience terrain. Elle sera difficilement une abstraction universelle, valable en tout temps et dans toute situation. Le deuxième corollaire est que cette théorie-de-l'action est potentiellement différente des discours officiels en fonction des exigences et des pressions extérieures: les justifications des actions et les discours de légitimation. Si le travail social se doit de comprendre son contexte et d'établir un dialogue avec les rationalités officielles des gouvernements et de leur emprise sur les services sociaux et sur les organismes subventionnaires, il se doit, aussi, de chercher sa propre voix, sa propre rationalité, qui sera le fondement de son autonomie.

\section{LA RECHERCHE D'UNE FORMATION NON BANCAIRE À LA THÉORIE}

Quelle est la pertinence du constructivisme dans la formation en travail social ? Nous pouvons en signaler deux aspects : il permet de critiquer les contraintes imposées par une idéologie positiviste dans la formation théo- 
rique des travailleurs sociaux, et il permet l'appel au respect intellectuel des étudiants en formation.

Les perspectives constructivistes, plutôt que de menacer la rationalité scientifique, peuvent élargir cette rationalité au-delà des frontières arbitraires qui lui sont posées par des logiques qui se pensent et se disent des logiques d'objectivation, mais qui sont en fait des logiques de légitimation. Le constructivisme peut ainsi éclairer la manière dont le travail social crée ses propres barrières en restant prisonnier de la sursocialisation de sa pensée. C'est par sa domestication aux discours dominants sur l'objectivité des sciences et de ses critères de sélection d'orientations et des méthodes, sur l'omniscience et l'impartialité sociale de l'État dans sa définition des problèmes sociaux et sur la pleine liberté des usagers potentiels dans la formulation de ses demandes que le travail social se prive de sa propre rationalité.

Si les perspectives constructivistes peuvent être classées comme des extensions opportunistes de la rationalité moderne, ou comme les excès irresponsables qui mènent à son écroulement, il y a un troisième angle, bienveillant celui-ci, qui signale son potentiel d'illumination du travail social. Si la raison instrumentale qui anime la Raison, l'État et l'Individu réduit le travail social à un contrôle social, il reste encore à expliquer "cette distance qui sépare l'intervenant de la fonction sociale qu'on voudrait lui assigner» (Renaud, 1990: 35). Cette distance, qui laisse de la place à la conviction morale, à l'option politique, à la solidarité et à I'altruisme doit être franchie et comprise; elle peut être comprise comme un travail sur le travail, comme l'effort des travailleurs du social pour valider leur travail par une compréhension élargie de la dynamique de la construction de sa rationalité - une rationalité qui ne serait pas menacée par l'incorporation à son entreprise de la solidarité, de l'altruisme et de l'espérance.

Cette récupération des rationalités permet de concevoir autrement le connaître de l'étudiant et de mieux le respecter comme théoricien de sa vie et de sa pratique future. Cela implique d'abord de reconnaître le fait de son autonomie intellectuelle.

[...] les élèves et étudiants, bien avant leur fréquentation des classes de sciences ou en marge de celles-ci, conceptualisent à leur façon les phénomènes de leur quotidien et tendent à maintenir ces conceptions, dites spontanées, tout au long de leur itinéraire scolaire, et cela, malgré l'enseignement des représentations formelles [scientifiques] de ces phénomènes (Larochelle et Désautels, 1992: 1).

L'apprentissage scientifique $n^{\prime}$ est plus compris comme le remplacement des formes "vulgaires" de compréhension par d'autres, dites scientifiques, mais plutôt comme un problème d'identification et de 
différenciation des contextes de signification, pour faire une place à la contribution des sciences:

[...] il ne s'agit plus de promouvoir la supériorité du savoir scientifique sur les autres savoirs, mais bien de le resituer au sein de la panoplie des jeux de connaissance inventés par les hommes et les femmes pour organiser, de façon viable, leurs expériences de cognition (Larochelle et Désautels, 1992: 4-5).

Si la théorie est toujours hantée par la légitimation que lui octroient les figures d'autorité, la formation théorique des intervenants fait face à un défi : faire comprendre que toute action professionnelle réfléchie et critique exprime une rationalité et, donc, une théorie larvée. Ce n'est certes pas une théorie en tant qu'explication complète, cohérente, définitive: $c^{\prime}$ est une théorie en construction, un produit de la situation qu'elle essaie de comprendre. Elle est enracinée dans une histoire qui est une tradition, légitimée par une autorité collective, toujours inséparable d'un contexte social dans lequel elle trouve son utilité, toujours appropriée par des personnes réelles, qui l'intègrent à leurs perspectives, à leurs intérêts et à leurs passions. Elle est mal représentée par les discours hérités ou empruntés, par I'utilisation d'un vocabulaire qui cache plus que ce qu'il dévoile. Les questions pertinentes dans la formation théorique ne renvoient pas à la mémorisation des discours, pas plus qu'elles ne renvoient à un simple remplacement des termes ou à une traduction d'un langage théorique à un autre. Invoquer la pyramide de Maslow, répéter l'escalier d'Erikson sont des invocations, non pas des théorisations. Parler d'acteurs sociaux et de rapports sociaux peut déguiser le fait qu'on parle d'individus et de relations interpersonnelles. On peut parler d'identité et penser individualité, parler d'autonomie et penser autosuffisance. Le vrai défi est de partager un sens de la théorie comme une élaboration tâtonnante des significations, un processus dynamique pour rendre conscient, visible, explicite un effort de comprendre une expérience. Tout comme l'action, la théorie est un mouvement difficile à délimiter dans le temps et à découper des autres significations construites par la même collectivité, sur l'arrière-plan d'une communauté de traditions et de destin.

En travail social, le constructivisme en est encore à ses balbutiements. Les travaux pionniers d'England, de Goldstein et de Scott commencent en puisant dans des courants plus développés, comme ceux qui parcourent l'éducation et la réflexion féministes. Les hypothèses de travail de Schön et de St-Arnaud sont en lien direct avec l'analyse du discours professionnel:

En généralisant les observations qu'ils ont faites sur des milliers de cas, ces deux auteurs ont formulé la conclusion suivante qu'on peut appeler la loi d'Argyris et Schön: 
Dans une situation difficile, il y a un écart systématique entre la théorie professée par le praticien pour expliquer son comportement et la théorie qu'il pratique à son insu, telle qu'on peut l'inférer à partir d'un dialogue réel (St-Arnaud, 1992: 53).

Les recherches épistémologiques sur l'enseignement des sciences de Nadeau et Désautels et de Larochelle et Désautels sont des voies de recherche prometteuses pour étudier la rationalité de l'action professionnelle. Les travaux de Lather et de Scott montrent les acquis épistémologiques des perspectives féministes, capables de faire une herméneutique constructiviste de la transmission bancaire des théories, "selon laquelle la seule marge de manœuvre qui s'offre aux élèves est celle de recevoir les dépôts, de les garder et de les archiver » (Freire, 1974: 51), même quand il s'agit de critiquer la transmission bancaire des théories critiques! Le dogmatisme des certitudes, l'autoritarisme intellectuel et la manipulation pédagogique ne sont malheureusement pas étrangers à l'enseignement des théories critiques (Lather, 1990).

Pour le travail social, le constructivisme illumine une perspective pédagogique ainsi qu'une ouverture épistémologique et politique. Pédagogiquement, il incite à construire avec les étudiants et les praticiens en formation leur compétence pour l'action - compétence active, critique et créatrice, autant intellectuelle que technique. Sa devise peut bien être: "toute pratique éducative ne peut faire l'économie du savoir des apprenants : il faut composer avec! » (Larochelle et Désautels, 1992: 33). Pour une profession qui travaille habituellement avec un sentiment $d^{\prime}$ être un instrument des centres de pouvoir et une conviction d'être proche des secteurs marginalisés, le constructivisme est une invitation à une pensée

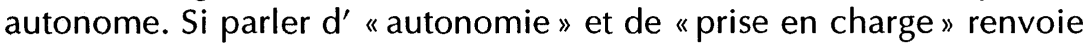
habituellement à l'action, le constructivisme rappelle que l'originalité de I'analyse et la libération de la pensée dans l'action sont les fondements $d^{\prime}$ une action professionnelle autonome et donc responsable.

On voit maintenant que la question posée au début de cet épilogue (quelle réalité le constructivisme construit-il ?) est fondamentalement fausse, mais aussi qu'il fallait faire cette erreur pour qu'elle se révèle comme telle. Le constructivisme $n^{\prime}$ invente pas ou n'explique pas une réalité indépendante de nous. II montre au contraire qu'il n'y a ni intérieur, ni extérieur, ni objet, ni sujet, ou plutôt que la distinction radicale entre sujet et objet - à l'origine de la construction d'innombrables " réalités » - n'existe pas, que I'interprétation du monde en fonction des paires de concepts opposés n'est qu'une invention du sujet, et que le paradoxe débouche sur l'autonomie (Watzlawick, 1988 : 354). 


\section{Références bibliographiques}

BLOOR, D. (1976). Knowledge and social imagery. Londres: Routledge \& Kegan Paul.

BERGER, P.L. et T. LUCKMANN (1967). The social construction of reality. A treatise in the sociology of knowledge. Garden City, NY: Doubleday.

BOUDON, R. et F. BOURRICAUD (1982). Dictionnaire critique de la sociologie, $2^{\mathrm{e}}$ éd. Paris : PUF.

BUNGE, M. (1991) "La botanique est venue après les fleurs », Philosopher, 11 : 41 51.

CAMPBELL, D.T. (1988a). "Evolutionary epistemology», dans D.T. CAMPBELL, Methodology and epistemology for social science, Selected papers. (E.S. Overman, ed.). Chicago: University of Chicago, p. 393-434.

CAMPBELL, D.T. (1988b). «Descriptive epistemology: Psychological, sociological and evolutionary ", dans D.T. CAMPBELL Methodology and epistemology for social science, Selected papers. (E.S. Overman, ed.). Chicago: University of Chicago, p. 435-486.

Claval, P. (1980). Les mythes fondateurs des sciences sociales. Paris: PUF.

ECO, U. (1980). Le nom de la rose. Paris: Grasset.

ECO, U. (1990). Le pendule de Foucault. Paris: Grasset.

ENGLAND, H. (1986). Social work as art. Making sense for good practice. Londres: Allen \& Unwin.

FREIRE, P.(1974). Pédagogie des opprimés, suivi de Conscientisation et révolution. Paris : Maspéro.

GADAMER, H.G. (1984). "What is practice? The conditions of social reason", dans H.G. GADAMER, Reason in the age of science. Cambridge, MA: The MIT Press, p. 69-87.

GeERTZ C. (1983). «Common sense as a cultural system», dans C. GeERTZ, Local knowledge. Further essays in interpretive anthropology. New York: Basic Books, p. 73-93.

GOLDSTEIN, H. (1990). "The knowledge base of social work practice: theory, wisdom, analogue, or art? ", Families in society: The journal of contemporary human services. vol. 71, $\mathrm{n}^{\circ} 1: 32-43$.

GOULDNER, A.W. (1970). The coming crisis of Western sociology. New York: Avon.

GUBA, E.G. et Y.S. LINCOLN (1989). Fourth generation evaluation. Newbury Park, $\mathrm{CA}$ : Sage.

HOLLIS, M. (1982). "The social destruction of reality», dans M. HOLLIS et S. LUKES, (dir.). Rationality and relativism. Cambridge, MA: The MIT Press.

KNORR-CETINA, K. (1981). The manufacture of knowledge. An essay on the constructivist and contextual nature of science. Oxford: Pergamon Press.

LAROCHELLE, M. et J. DÉSAutels (1992). Autour de l'idée de science. Itinéraires cognitifs d'étudiants et d'étudiantes. Sainte-Foy, QC: Université Laval / De Boeck Wesmael.

LATHER, P. (1991). Getting smart. Feminist research and pedagogy within the Postmodern. New York: Routledge. 
LATHER, P. (1990). "Staying dumb? Student resistance to liberatory curriculum ». American Educational Research Association, Boston, p. 16-20.

LATHER, P. (1990). "Postmodernism and the human sciences », The humanistic psychologist, vol. 18, $\mathrm{n}^{\circ} 1: 64-84$.

LEFRANÇOIS, R. (1990). "Lecture de la recherche sur les problèmes sociaux", Service social, vol. 39, $\mathrm{n}^{\circ} 2: 129-139$.

LYOTARD, J-F. (1979). La condition postmoderne. Rapport sur le savoir. Paris: Les Éditions de Minuit.

MAO TSE TOUNG (1972). "D'où viennent les idées justes? ", dans Textes choisis de Mao Tse Toung. Pékin : Éditions en langues étrangères, p. 544-546.

MASTERMAN, M. (1974). "The nature of a paradigm ", dans I. LAKATOS et A. MUSGRAVE (dir.) Criticism and the growth of knowledge. Londres: Cambridge University, p. 59-89.

MAturana, H. et G.F. VAREla (1984). El árbol del conocimiento. Las bases biológicas del entendimiento humano. Santiago, Chili : Editorial Universitaria.

NADEAU, R. (1991). "Trois images de la science ", Philosopher, $11: 21-39$.

NADEAU, R. et J. DÉSAUTELS (1984). Épistémologie et didactique des sciences. Ottawa: Conseil des sciences du Canada.

NISBET, R.A. (1969). Social change and history. Aspects of the Western theory of development. New York: Oxford University Press.

POLANYI, M. (1964). Personal knowledge. Towards a post-critical philosophy. New York: Harper \& Row.

POLANYI, M. (1966). The tacit dimension. Garden City, NY: Doubleday.

QUÉBEC, GOUVERNEMENT DU (1980). Un projet collectif. Énoncé d'orientations et plan d'action pour la mise en œuvre d'une politique québécoise de la recherche scientifique. Québec: Éditeur officiel.

RACINE, P. (1991). "L'usage des théories de l'action dans la formation à l'intervention sociale», Service social, vol. 40, $n^{\circ} 2: 7-25$.

RENAUD, G. (1990). "Travail social, crise de la modernité et post-modernité", Revue canadienne de service social, vol. 7, $\mathrm{n}^{\circ} 1: 27-48$.

SCHÖN, D.A. (dir.) (1991). The reflective turn. Case studies in and on educational practice. New York, NY: Teachers College.

SCHÖN, D.A. (1987). Educating the reflective practitioner. San Francisco, CA: Jossey-Bass.

SCHÖN, D.A. (1983). The reflective practitioner. How professionals think in action. New York: Basic Books/Harper Colophon.

SCHUTZ, A. (1962). "Common-sense and scientific interpretation of human action », dans A. SCHUTZ, Collected papers, vol. 1. La Haye: Martinus Nijhoff, 3-47.

SCHUTZ, A. (1962). "Equality and the meaning structure of the social world", dans A. SCHUTZ, Collected papers, vol. 2. La Haye: Martinus Nijhoff, 226273.

SCHUTZ, A. et T. LUCKMANN (1973). The structures of the life-world. Evanston, IL: Northwestern University.

SCOTT, D. (1989). "Meaning construction and social work practice", Social Service Review, March, 39-51. 
ST-ARnAud, Y. (1992). Connaître par l'action. Boucherville, Québec: Presses de I'Université de Montréal.

VON GLASERSFELD, E. (1988). "Introduction à un constructivisme radical ", dans P. WATZlAWICK (dir.) L'invention de la réalité. Contributions au constructivisme. Paris: Seuil, p. 19-43.

WATZlAWICK, P. (1988). "Épilogue», dans P. WATZLAWICK (dir.) L'invention de la réalité. Contributions au constructivisme. Paris: Seuil. 